\title{
SRST: A Storytelling Model Using Rhetorical Relations
}

\author{
Arturo Nakasone and Mitsuru Ishizuka \\ Dept. of Information and Communication Engineering, University of Tokyo, 7-3-1 Hongo, \\ Bunkyo Ku, Tokyo 113-8656, Japan \\ arturo@mi.ci.i.u-tokyo.ac.jp, ishizuka@i.u-tokyo.ac.jp
}

\begin{abstract}
Storytelling models are usually constrained to the applications they are implemented in because of the particular characteristics of the data used to define story events and the way those events are linked. In order to develop a more generic model to create storytelling applications, we need to focus the solution not on the data itself, but on the manner this data, in the form of events, is organized and conveyed to the user. In this paper, we present SRST (Storytelling RST), our proposal for a generic storytelling ontology model based on the organization of events using the relations proposed by the Rhetorical Structure Theory (RST) and how narrative principles are applied to these RST relations to generate coherent stories.
\end{abstract}

Keywords: Storytelling, Ontology Model, RST, Rhetorical Relations.

\section{Introduction}

In a broad sense, stories are defined as "unique sequences of events, mental states, or happenings involving human beings as characters or actors" [4]. The notion of story event is defined in several ways, each one to suit a particular way to deliver the intended message to the audience. Events can be represented as plain text $[8,26,28]$, scripted individual character actions [9], story world states [14,15,18,30], or multimedia content $[5,23,31]$. A storytelling model is usually constrained to a particular application due to the nature of these events and the way they are linked to one another. Most applications rely on the content of the event to describe a story, and its inherent temporal property to construct it. Nevertheless, this tight coupling between content and temporality makes it very difficult to use or even adapt the model to other domains. In addition, most applications rely on the implicit temporal relation of events to convey a narrative experience, but in most cases, this experience is predetermined, either by defining event by event sequences or by grouping events with similar space and temporal attributes and defining group by group sequences. In order to develop a more generic model to create storytelling applications, we need to focus the solution not on the content itself, but on the manner this content, in the form of events, is organized and how this organization can be conveyed to the user in the context of a narrative experience.

In this paper, we present SRST (Storytelling RST), our proposal for a generic storytelling ontology model based on the organization of events using the relations proposed by the Rhetorical Structure Theory (RST) [16] and narrative principles 
applied to these RST relations. This model was developed as an extension to our web presentation system [20].

The rest of the paper is organized as follows. The next section will present related work on storytelling applications from the point of view of events. Section 3 discusses our approach to a generic storytelling model based on ontology classes and narrative rules. Section 4 presents the current implementation of our web based application that uses annotated data from the ontology. Finally, our plans for future research topics and a summary will conclude the paper.

\section{Related Work}

Even though storytelling applications were mostly oriented to text generation in the beginning, the range of applicability of storytelling has increased due to the ubiquitous presence of communication networks such as the Internet, and the availability of multimedia content. Most researchers assume a concept of "event" in order to organize the content of their applications, and, therefore, have developed storytelling engines to deliver such events using narrative techniques. Nevertheless, the algorithms implemented in those engines were selected because of the event definition they implied and vice versa.

State Transition based applications [9,14,18,26,29] define events as states that specify the current situation in a particular point of the story. Bayesian Networks, Finite State Machines, and their variations are commonly used as storytelling engines. Therefore, events must be annotated to fit the requirements for nodes in the network or machine.

In Goal based applications [6,22,32], a goal event or events are established as the final outcome of the story. From a set of initial conditions, a story is unfolded by the sequence of events that are needed to reach such goal event or events. Planners and their variations are commonly used for these applications. Due to the nature of planners, events have to be annotated with a set of pre and/or post conditions. Nevertheless, some applications make use of special narrative functions that enforce narrative principles in the context of the whole story [17,30]. Therefore, event sequencing is constrained not only by direct event links, but also by each event contribution to the overall storytelling experience.

In Template based applications [10,27,31], events are selected to fit story templates and permutated to create new narrative experiences based on those templates. Since the story template constrains which and how events are presented, these events must be annotated to determine their role in the context of the whole template. Script based applications $[1,23,25,28]$ are a particular case in which stories are described using a high level language. The application, then, present the events in the way specified by such scripts. In some cases, narrative or dramatic effects are applied during the event transitions.

Semantically organized events using primarily RST have been used in applications that present multimedia content $[5,11,13]$, but most of them use only a very limited set of relations. Since rhetorically structured content was mainly used for organizational purposes, these applications do not deal with the narrative implications that rhetorical relations have in storytelling-like presentations. 
Even though the results obtained in these applications are impressive from the storytelling point of view, the cohesion between event definition and storytelling engine makes the task of adapting these models to other domains extremely difficult. Therefore, a model in which event definition and sequencing are separated is necessary to guarantee its generic attribute.

\section{A Semantic Approach to Storytelling}

Events in the world are not isolated but interconnected through some kind of relation. Even though each event itself is meaningful in its content, the relations between them are what make them meaningful in the context of a story. Semantic organization gives us not only a relationship of meaning, but also a relationship of temporality through the use of rhetorical extrapolations. This organization gives us enough flexibility to create stories, regardless of the kinds of events used.

In this section, we will present our approach to define a general ontology model for storytelling based on semantic RST relations. We will define not only how RST relations are referenced in the context of stories, but also how narrative properties are enforced by the proper use of these relations.

\subsection{Story Concepts - Defining What a Story Is About}

Even though stories are commonly referred to as "sequences of events", they are always developed in some particular context or around a particular concept. When we talk about stories, we need to convey to the user not only a general sequence of isolated events, but also a series of interconnected ideas that will evolve as a knowledge pattern inside our memories. Using storytelling to convey this complex mesh of ideas is what gives storytelling methods and properties their true value [7]. Therefore, to properly address this issue, our generic ontology must support a concept representation model that can reflect not only this complex organization of ideas but also a way to convey them through narrative channels.

In our model, a Concept Ontology is defined as a networked organization of nodes, which are connected through directed links. Each node is defined as a Concept or "a particular theme a story or part of it may talk about", and each link is defined as "a directed relation that defines the dependency between the two connected concepts".

When a story is fully constrained to talk about one particular topic, only one node in the Concept Ontology is referred; but when a story spans several topics, a smooth transition through the use of links between concepts ensures a fluid narrative experience. Even though links only specify a requirement relation between two concepts (i.e. the telling of one concept must precede the telling of the other), it also gives a pseudo-temporal relation since concepts are conveyed through linear narrative channels.

The main advantage of this model is that this sequenced organization of issues allows users to construct fluid and coherent stories based on the selection of a few key concepts. When a user specifies the key issues of the story, the storytelling model engine automatically selects other concepts that must be included in order to have a fluid experience. 


\subsection{Rhetorical Relations - Defining How a Story Is Organized}

Events are related to one another with some kind of relation. From the very definition of story, we can extract the most common one: diachronicity [4], which means that events have a temporal relation between them (i.e. one event happens before, at the same time, or after another). In most cases, this attribute is taken for granted in any storytelling application and researchers have tried to enrich this temporal synchronization of events by modifying the mechanisms by which events are put one after the other.

Nevertheless, temporal relations are only one aspect in storytelling. Event temporization refers to the fact that narrative channels of communication are linear and, therefore, the only way to transmit these events is through the use of a timesequenced pattern. In fact, stories in our minds are much more than a linear definition of events. Stories are "complete patterns that communicate a special kind of knowledge to our pattern recognizing mental module" [7]. Since these patterns are present in our minds as a web of interconnected events, it is clear that such connections deal with much more complex relations than temporal ones.

Natural text is one of the oldest ways to transmit a story and has been extensively analyzed in order to discover which rules govern its generation not only in terms of text organization, but also in terms of narrative characteristics. From a semantic point of view, rhetorical theories have provided us with the most useful analysis on which kinds of relations can be defined between pieces of text in a narrative. Moreover, it can be stated that rhetorical relations reflect not only semantic relations between pieces of text, but also between ideas, concepts, and events in a broader sense. Relations such as CAUSE (i.e. one event is the cause of another) or BACKGROUND (i.e. one event serves as background information for the other) that can be inferred from a text reflects not only the organization of those pieces of information in the text, but also the meaning that those events have in the mental story pattern of the author of such text.

In order to accurately reflect this story pattern in our model, we defined the relations between events as rhetorical. By defining only the relations between events and not the events themselves, we were able to enforce the generic attribute of the storytelling ontology model.

\subsection{SRST: The Storytelling Ontology Model}

Taking into consideration the conceptual and semantic aspects described above, we propose an ontology model implemented using OWL [21] that deals with the generic aspects of the storytelling construction process. The classes in this model were defined considering the many different definitions that researchers gave to their story components, but associating each class with a more general meaning that encompasses all those different definitions. Each class has a purpose in the context of story pattern organization, either to define a specific story component or a property of such component. The classes defined for the present version of SRST are:

- Concept: A Concept defines a specific topic that a story or part of it may refer to.

- Event: An Event is defined as a single piece of meaningful information worthy of being shown. Due to the generic property of SRST, an Event can specify a reference to piece of text, video clip, image, game scene, character scripts, etc. 
- Relation: A Relation is a rhetorical binding between two entities, which states a specific rhetorical function. As specified in RST, entities in a Relation can be both Nucleuses (which is defined as a Multinuclear Relation Type), or a Nucleus - Satellite pair (which is defined as a Nucleus-Satellite Relation Type)

- Act: An Act is defined as a hierarchical structure composed of Nucleus and Satellite entities, joined by Relations. This class describes the minimum level of story organization in which a story may arise. An Act is a recursive structure, which means that Nucleus and/or Satellite entities can represent an Event or another Act object.

- Scene: A Scene is defined as a set of Acts, which are grouped in the context of a single Concept.

- Agent: An Agent is an actor that takes part in a Scene by executing or being part of one or more Events.

- Role: A Role is a part that an Agent plays during a Scene.

In the next subsections, we will explain the relations among the SRST classes and how these relations contribute to the overall story organization. The complete ontology model diagram is shown in Figure 1.

Scenes and Acts. In SRST, we made a clear distinction between semantically organized events (Acts) and conceptually organized events (Scenes). Even though they are indivisible properties for any story, this separation has been established to emphasize the fact that conceptually organized events can have different semantic organization to express, either the same content or a different content related to the same concept. For instance, if we were to define the Concept "ARRIVING LATE TO WORK", we could express the idea of this concept by using these two different semantic organizations of events:

- It was raining, THEREFORE, I missed the train (CONSEQUENCE relation)

- I missed the train, BECAUSE it was raining (CAUSE relation)

Even though we used the same set of events in this case, the relations used to join them are different and, therefore, the emphasis on which event is more important from a user's perspective changes accordingly.

Acts, Nucleuses, and Satellites. An Act represents a basic structure in which nucleus and satellite components are connected through relations. Even though SRST is flexible enough to allow an unlimited number or nucleus and satellite components connected to each other, in our current implementation, this structure is constrained to allow the definition of: (a) purely multinuclear relations of the same type, or (b) a single nucleus with one or more satellites with the same or different types or relations. This is due to the fact that we are primarily dealing with events in the form of texts to be uttered by an Agent. As more narrative domains are analyzed, other combinations in the Act structure will be implemented.

Scenes, Agents, and Roles. In every Scene, Agents are defined as character entities that either present the content of Events or take part during the execution of Events as actors. Since not every Agent can be part of every Event inside a Scene/Act, the Role 


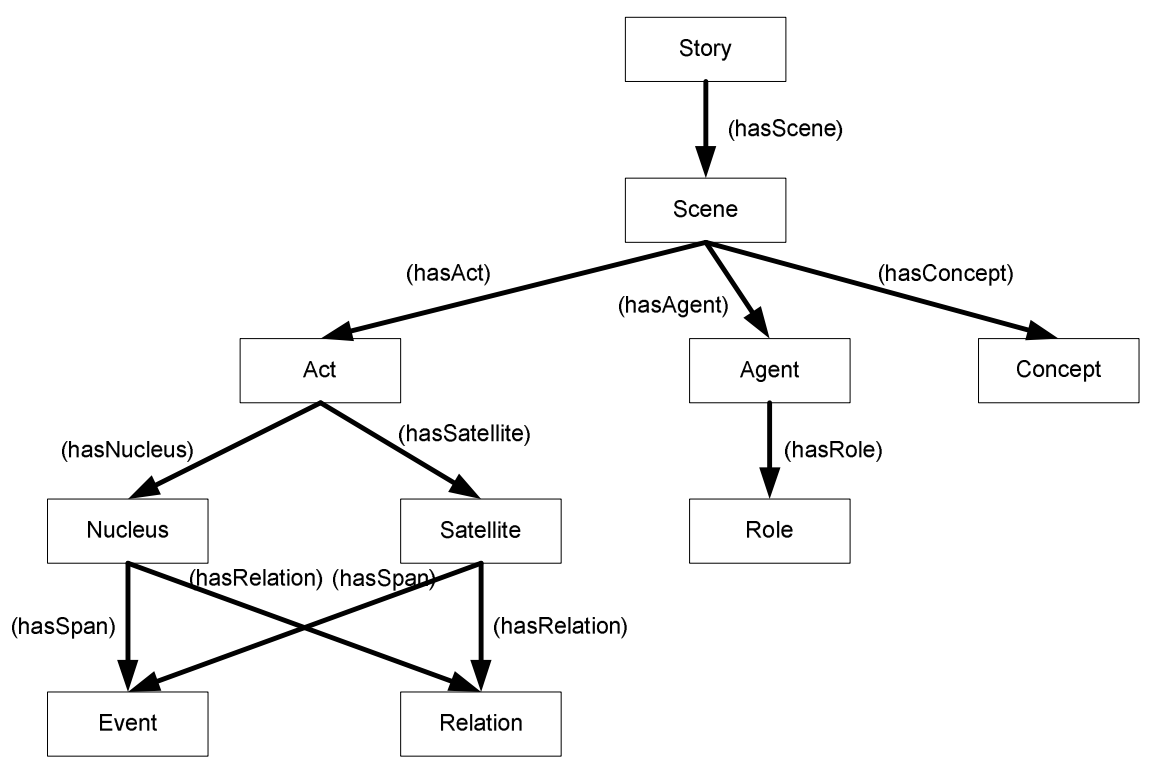

Fig. 1. The Complete Storytelling Ontology Model (SRST) based on RST relations

object is associated with every Agent and defines in which kind and on which side of relations it may intervene. Although Roleobjects depend on the kind of application in which the ontology is used, we have predefined five roles that identify and group the main characteristics of our RST relation set:

- Questioning Role: The Agent represents the character to which the information contained in the relation will be conveyed to. (e.g. Nucleus of SOLUTIONHOOD)

- Informing Role: The Agent represents the character that will convey the information contained in the relation (e.g. Satellite of SOLUTIONHOOD)

- Contrasting Role: The Agent represents a character that contrast information of one side of the relation with another (e.g. Satellite in CONTRAST)

- Convincing Role: The Agent represents a character that tries to gives a convincing explanation about the information contained in a relation (e.g. CAUSE)

- Evaluating Role: The Agent represents a character that states a final conclusion or assessment in a relation (e.g. EVALUATION)

We have defined the most important elements and their relations for any storytelling application. In the next section, we will define the rules by which these elements interact in order to assess narrative quality.

\subsection{Adding the Narrative Component to SRST}

The narrative rules defined in SRST are based on Jerome Bruner's work on narrative and its characteristics [4]. Even though SRST deals with the majority of Bruner's 
narrative principles to some extent, we will concentrate on the most important aspect of narrative: how events shall be put together in order to constitute a narrative, which is known as the principle of Hermeneutic Composability. The concepts and formulas proposed for SRST express the point of view of the authors on how Bruner's principles can be implemented in a computer model using RST, and should not be taken as a definite implementation guide. In future versions of the model, we will refine our assumptions using more accurate empirical information.

To address this principle, we have identified three stages in which story construction takes place: (a) Event Selection inside an Act, (b) Act Selection inside a Scene/Concept, and (c) Scene/Concept Selection in a Story.

Event Selection inside an Act. This is the lowest level of the SRST event organization scheme in which a story arises. In this level, our definition of story construction was centered in the concept of Conflict [3], defined as an imminent change on the current state of affairs. Based on this concept of Conflict, we classified our set of RST relations in:

(a) Relations that may introduce a Conflict or Conflict Relations (CONTRAST, SOLUTIONHOOD, ELABORATION, CONSEQUENCE, and SEQUENCE). Since not every Conflict Relation has the same potential to arise a Conflict, a numerical value known as the Conflict Weight Value (CWV) was assigned to each of these relations to show their importance in terms of conflict potential. The range of the $\mathrm{CWV}$ goes from 0 to 1 , with higher values indicating higher conflict potential.

(b) Relations that provide the events to successfully resolve the conflicts or Resolution Relations.

Even though a general story is constructed around a Conflict and its resolution [3], a proper order of Conflict and Resolution relations must be defined in order to create narrative tension [17]. In SRST, we defined this order based on the assumptions that:

- Relations with low CWV build less narrative tension than relations with high CWV.

- Context and content explanations should precede results and user belief alterations to build up narrative tension.

Therefore, for each story constructed inside the scope of an Act, the following relations are to be considered in this specific order when available:

- Background Information

- Conflict Presentation. According to the CWV for Conflict Relations, conflicts will be created in a way that weaker conflict relations will be presented first.

- Resolution of Conflict, which is achieved when the information on the other side of the relation is stated. In order to build up narrative tension, relations are to be chosen following this pattern:

- Context Explaining Nodes: Circumstance, Purpose

- Multinuclear / Temporal Nodes (Content Explaining Nodes): List, Temporal_Before,Temporal_SameTime,Temporal_After 
- Result Nodes: Result, Cause

o Presentational Nodes (User's belief alteration nodes): Evidence, Enablement

- Restatement or Evaluation. If both are available, the Evaluation relation will come first, since it is assumed to contain more narrative tension that the Restatement relation

Act Selection inside a Scene/Concept. Similar to the Event Selection case, Acts inside a Scene are selected in a way that they build narrative tension through the presentation of all the Acts in the Scene. To determine the narrative tension degree for an Act, we have defined two parameters that measure its tension level:

- Conflict Resolution Value (CRV): The CRV value is calculated based on the number of possible conflicts in the different levels of the Act structure. Conflicts that arise in higher levels of the Act structure have greater value than those that arise in lower levels. Therefore, the CRV for an Act is defined as a measure of conflict potentiality and is calculated through the following formulas:

$$
\begin{gathered}
C R V(A)=C_{0}(A)+\sum_{j=1}^{n-1} \frac{C_{j}(A)}{2 j} . \\
C_{j}(A)=\sum_{k=1}^{5} C W V_{k} \times N_{k}(j) .
\end{gathered}
$$

Where:

- $\mathrm{CRV}(\mathrm{A})$ is the CRV for the Act

- $\mathrm{C}_{0}(\mathrm{~A})$ is the number of conflict relations in the first level of the Act structure $^{1}$

- $C_{j}(A)$ defines a weighed value based on the total number of conflict relations in the $\mathrm{j}^{\text {th }}$ level. In this formula, the total number of conflicts in each conflict relation (five in total) is multiplied by its corresponding CWV.

- $\mathrm{n}$ is the maximum number of levels in the Act structure

- $\mathrm{CWV}_{\mathrm{k}}$ is the Conflict Weight Value for a particular type of Conflict $\mathrm{k}$.

○ $\mathrm{N}_{\mathrm{k}}(\mathrm{j})$ is the number of conflicts of type $\mathrm{k}$ in the $\mathrm{j}^{\text {th }}$ level

- Multinuclear Relations Value (MRV): The MRV value is calculated based on the number of multinuclear relations in several levels of the Act structure. Multinuclear relations in higher levels have greater value than those deeper in the hierarchy. Therefore, the MRV for an Act is calculated through the following formula:

${ }^{1}$ Given the recursive nature of the Act structure, the level value was defined to specify how deep an Act structure goes into such recursion. A zero level Act structure indicates that it is not contained in any other Act structure. Likewise, $a \mathrm{j}^{\text {th }}$ level Act structure indicates that it is contained in $\mathrm{j}$ Act structures. 


$$
\operatorname{MRV}(A)=N_{0}(A)+\sum_{j=1}^{n-1} \frac{N_{j}(A)}{2 j}
$$

Where:

- $\operatorname{MRV}(\mathrm{A})$ is the MRV for the Act

- $N_{0}(A)$ is the number of nucleuses in the first level of the Act

- $\quad \mathrm{N}_{\mathrm{j}}(\mathrm{A})$ is the total number of nucleuses in the $\mathrm{j}^{\text {th }}$ level

O $\mathrm{n}$ is the maximum number of levels in the Act structure

To create narrative tension during the Act Selection process, it was assumed that multinuclear relations (e.g. SEQUENCE, LIST) inside an Act have more conflict potential due to the possibility of contrast between their components. Therefore, the order in which Acts are to be selected inside a Scene was defined by the following criteria:

- Acts with Background Information must be selected first when available. This criterion was set to satisfy Bruner's Referentiality principle [4].

- Acts with low MRV are selected before Acts with higher MRV. This ensure a narrative tension build up inside the Scene.

- If two Acts have the same MRV, the Act with the lowest CRV is selected first.

Scene/Concept Selection inside a Story. In SRST, a Story is constructed by selecting which Concepts from the Concept Ontology are to be included in it. Once the Concepts are selected, SRST calculates the optimum path through the ontology that guarantees a smooth transition between all the selected Concepts.

In extreme cases, if no path is found, selected Concepts are treated as individual stories. Therefore, it will depend on the author of the story to ensure a good narrative experience to his or her audience by providing a well defined concept ontology.

\section{Current Implementation}

The application to test our ontology model was implemented using .NET technology, Visual C\#, and Java. It consists of 3 main modules:

Text Services: The Text Services Module is the module in charge of dealing with text processing and format conversions into the input needed by the system. Since creating the OWL text data can be a cumbersome process, this module facilitates the work of creating these files by processing LISP formatted files obtained with the RSTTool [24]. Even though the RST annotation process must still be done with this tool, the conversion process into OWL files is greatly simplified with this module.

Visual Services: This module deals with the presentation of the OWL annotated content and acts as the interface of the application.

OWL Services: This module acts as a proxy between the OWL Reasoner module (in this version of the application, a reasoner called Kaon2 [12] has been used due to its 
simple interface) and the Visual Services module. The OWL Services module is in charge of loading the ontology definitions and data into the reasoner, calculate the CRV and MRV values for the ontology data, and retrieve events based on the narrative rules specified by the ontology. Since this module maintains the whole status of the story at any moment, it can be deployed using any visual interface with which can establish a socket connection.

The complete application design diagram is shown in Figure 2.

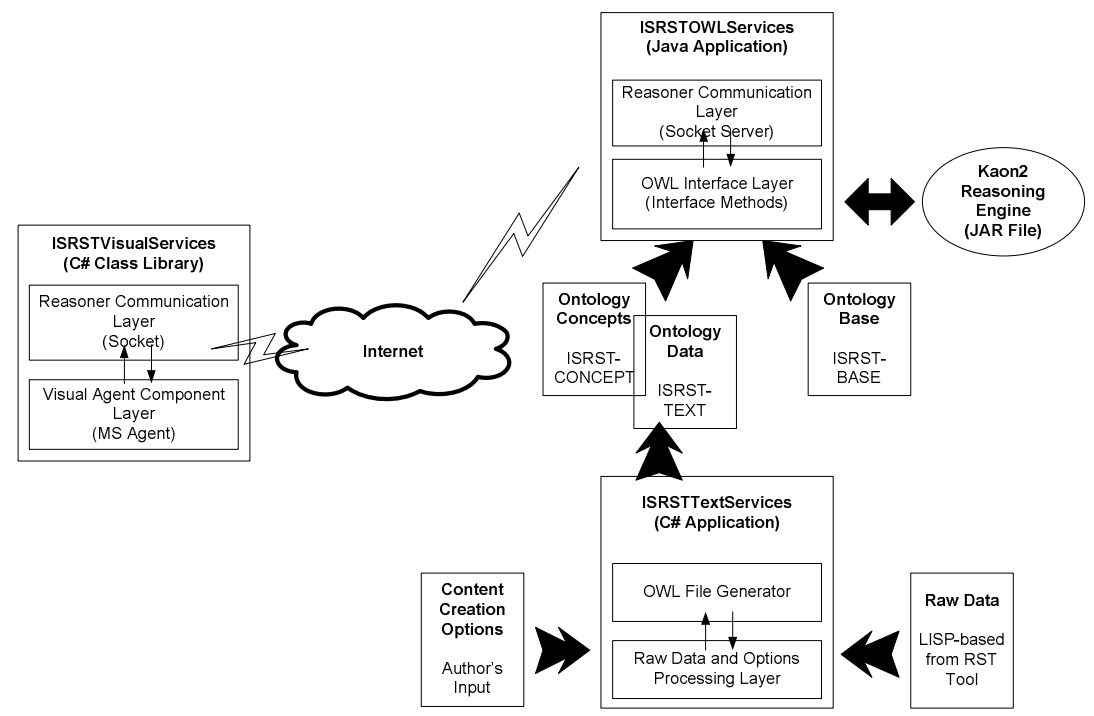

Fig. 2. Application Design Diagram

\section{Conclusion and Future Work}

In this paper, we have presented our proposal for a generic storytelling ontology model based on the organization of events using the relations proposed by the Rhetorical Structure Theory (RST). Even though the model was designed as generic as possible and taking into account all the different contributions from other works, there are some other elements that are part of stories that were not considered for this first version. Elements such as Location or Stage (see [10]), and Props (see [7]) will be analyzed to see how their contribution affects the narrative properties of the model.

Aside from making a complete ontology model for storytelling, our research is also focused on the interactive aspect of it. Therefore, our most immediate concern is to improve this model by analyzing how a user model can be implemented into this generic framework. Although most user model information depends on direct input received by the storytelling applications, our analysis will concentrate mostly on interaction that is non intrusive, namely, emotion recognition [19] and visual attention processing [2]. 


\section{References}

1. André, E., Concepcion, K., Mani, I., van Guilder, L. Autobriefer: A System for Authoring Narrated Briefings. Multimodal Intelligent Information Presentation, Oliviero Stock and Massimo Zancanaro, 143-158, Springer, 2005

2. Bee, N., Prendinger, H., Nakasone, A., André, E., and Ishizuka, M. AutoSelect: What You Want Is What You Get. Real-time processing of visual attention and affect. Proceedings International Tutorial and Research Workshop on Perception and Interactive Technologies (PIT-06), Springer LNAI 4021, Kloster Irsee, Germany, 2006, 40-52.

3. Bordwell, D. Narration in the Fiction Film. London Routledge, 1986

4. Bruner J. Acts of Meaning, Cambridge, Harvard University Press, 1991.

5. Callaway, C., Not, E., Novello, A., Rocchi, C., Stock, O., Zancanaro, M. Automatic Cinematography and Multilingual NLG for Generating Video Documentaries. In Artificial Intelligence, vol. 165, June 2005, 57-89

6. Cavazza M., Charles F., and Mead S.J. Interacting with virtual characters in interactive storytelling. In Proceedings First Conference on Autonomous Agents and Multiagent Systems (AAMAS-02), New York, 2002. ACM Press, 318-325

7. Crawford, C. Chris Crawford on Interactive Storytelling. New Riders 2005.

8. Figa, E. and Tarau, P. Story Traces and Projections: Exploring the Patterns of Storytelling. In N. Braun and U. Spierling, editors. First International Conference on Technologies for Interactive Digital Storytelling and Entertainment (TIDSE 2003), Darmstadt, Germany, March 2003.

9. Gebhard, P., Kipp, M., Klesen, M., Rist, T.: Authoring scenes for adaptive, interactive performances. The Second International Conference on Autonomous Agents and Multiagent Systems (AAMAS-03). 725-732

10. Gervas P., Diaz-Agudo B., Peinado F, and Hervas R. Story plot generation based on CBR. Journal of Knowledge Based Systems 18 (2-3), 2005, 235-242.

11. Geurts J., Bocconi S., van Ossenbruggen J., and Hardman L. Towards Ontology-Driven Discourse: From Semantic Graphs to Multimedia Presentations. In Proceedings of the International Semantic Web Conference 2003, 597-612.

12. Kaon2 Reasoning Engine. http://kaon2.semanticweb.org/

13. Little, S., Geurts, J., Hunter, J. Dynamic Generation of Intelligent Multimedia Presentations through Semantic Inferencing, In Proceedings of the 6th European Conference on Research and Advanced Technology for Digital Libraries, September 2002.

14. Magerko, B. and Laird, J.E. Building an Interactive Drama Architecture. First International Conference on Technologies for Interactive Digital Storytelling and Entertainment, 2003. Darmstadt, Germany, 226-237.

15. Magerko, B. and Laird, J.E. Mediating the Tension Between Plot and Interaction. AAAI Workshop Series: Challenges in Game Artificial Intelligence, 2004. San Jose, California, pp. 108-112.

16. Mann, W., and Thompson S. Rhetorical structure theory: A theory of text organization. Marina del Rey, CA: Information Sciences Institute, 1987.

17. Mateas, M., Stern, A. Façade: An Experiment in Building a Fully-Realized Interactive Drama; Game Developers Conference, Game Design Track, March 2003

18. Mott, B., Lester, J. U-DIRECTOR: A Decision-Theoretic Narrative Planning Architecture for Storytelling Environments. In Proceedings of the 5th International Joint Conference on Autonomous Agents and Multiagent Systems (AAMAS-2006), Hakodate, Japan, 2006 
19. Nakasone A., Prendinger H., and Ishizuka M. Emotion recognition from electromyography and skin conductance. The Fifth International Workshop on Biosignal Interpretation (BSI05), Tokyo, Japan, 2005, 219-222.

20. Nakasone A., Prendinger H., and Ishizuka M. Web Presentation System using RST Events. The Fifth International Conference on Autonomous Agents and Multiagent Systems (AAMAS-06), Hakodate, Japan, 2006, 995-997.

21. OWL - Web Ontology Language http://www.w3.org/TR/2004/REC-owl-features-20040210/

22. Riedl, M.O., Saretto, C.J., Young, R.M. Managing interaction between users and agents in a multi-agent storytelling environment. The Second International Joint Conference on Autonomous Agents \& Multiagent Systems, AAMAS 2003, July 14-18, 2003, Melbourne, Victoria, Australia, Proceedings. ACM 2003. 741-748

23. Rocchi, C., Zancanaro, M. Rhetorical Patterns for Adaptive Video Documentaries. In Proceedings of Adaptive Hypermedia and Adaptive Web-Based Systems AH 2004, Eindhoven, August 2004.

24. RST Annotation Tool. http://www.isi.edu/ marcu/

25. Sgouros, N.M., Papakonstantinou, G., Tsanakas, P. Dynamic Dramatization of Multimedia Story Presentations. ACM International Conference on Intelligent User Interfaces (IUI97), Orlando, FL, USA

26. Silva A., Guilherme R., and Paiva A. Tell me that bit again... Bringing Interactivity to a Virtual Storyteller. In Proceedings of the 2nd International Conference on Virtual Storytelling - ICVS 2003, 146-154.

27. Sobral, D., Machado, I., Paiva, A. Managing authorship in plot conduction. In Virtual Storytelling: Using Virtual Reality Technologies for Storytelling. Springer 2003.

28. Sumi, K., Tanaka, K. Automatic Conversion from E-content into Animated Storytelling, Entertainment Computing-ICEC2005, Springer Lecture Note in Computer Science, 24-35 (September, 2005).

29. Swartout, W., Hill, R., Gratch, J., Johnson, W.L., Kyriakakis, C., Labore, K., Lindheim, R., Marsella, S., Miraglia, D., Moore, B., Morie, J., Rickel, J., Thiebaux, M., Tuch, L., Whitney, R. Toward the Holodeck: Integrating Graphics, Sound, Caracter and Story in Proceedings of the 5th International Conference on Autonomous Agents, 2001

30. Szilas, N. IDtension: a narrative engine for Interactive Drama; 1st International Conference on Technologies for Interactive Digital Storytelling and Entertainment (TIDSE 2003), Germany, March 24-26 2003.

31. Wolff A., Mulholland P., and Zdrahal Z. Scene-Driver: A Narrative-Driven Game Architecture Reusing Broadcast Animation Content. In Proceedings of the Working Conference on Advanced Visual Interfaces, AVI 2004, Italy, 94-97.

32. Young, R.M. An Overview of the Mimesis Architecture: Integrating Intelligent Narrative Control into an Existing Gaming Environment. Working Notes of the AAAI Spring Symposium on Artificial Intelligence and Interactive Entertainment, Stanford, CA, March 2001 